\title{
Theoretical calculation model of the propulsion engine admission parameters for different functional conditions
}

\author{
Vlad Vornicu*, Tudor Ulian, Edward Rakosi and Sorinel Talif \\ "Gheorghe Asachi" Technical University of Iasi, Faculty of Mechanical Engineering, Department of \\ Mechanical Engineering and Automotive Engineering, Romania
}

\begin{abstract}
Inlet pressure as an engine parameter has a decisive role in the effective power and torque. Spark ignition engines achieve the load adjustment by changing the shutter position, which represents a quantitative adjustment. Obtaining a theoretical model to demonstrate the dependence between the engine speed and load will make possible an optimization of the combustion process.
\end{abstract}

\section{Introduction}

The pressure at the end of the intake stroke, currently denoted $p_{a}$, represents the pressure of the mixture between the fresh load and residual gasses from the cylinder at the end of the intake stroke when the cylinder gasses volume is maximum. This pressure value affects other parameters of the characteristic operating cycle for internal combustion engines, with an impact on the overall performance, by influencing the effective power, effective torque and the specific fuel consumption. This article presents a calculation model for $p_{a}$ pressure, developed by the authors, which takes into account, different from other known models, the load and engine speed modification, during a variation of the powered vehicle operating mode. In this way, the vehicle speed, fuel consumption or engine economic regime can be more precisely estimated [1].

\section{Proposed Theoretical Model}

In the context of this model, it is considered that the efficiency of cylinder filling, commonly referred by many authors as filling ratio, defined according to the literature as the charge amount of fresh mixture ratio effectively retained into the engine cylinder and also by considering the gasodynamic and thermal losses, it's not affected by the engine speed variation, but it is affected by the engine load, main consideration for spark ignition engine.

This consideration can be revealed by the following expression:

$$
\eta_{v} \neq f(n) \text {. }
$$

\footnotetext{
* Corresponding author: vladd.alex@yahoo.ro
} 
By taking into consideration the recommended values for $\eta_{v}$ on full load [2], and also experimental results [3], it was subsequently adopted a filling coefficient $\eta_{v}=0.87$.

Further, the situation of $p_{a}$ intake pressure variation was developed, at the same time with the engine load modification. In order to do this, one of the $p_{a}$ intake pressure expressions, recommended by some authors, was adapted, by introducing the amendment of filling coefficient modification, dependent on the engine load, denoted with $x$.

In this way:

$$
p_{a}(\chi)=\frac{p_{0}}{T_{0}} \cdot T_{a} \cdot\left(1-\varepsilon^{-1}\right) \cdot\left(1-\varphi_{a f}+\gamma_{r}\right) \cdot \eta_{v}
$$

By introducing further $\Phi_{0}\left(p_{0}, T_{0}\right)=\frac{p_{0}}{T_{0}}$, as a parameter which defines the environmental conditions, regarding pressure and temperature, the equation (2) becomes:

$$
p_{a}(\chi)=\Phi_{0} \cdot T_{a} \cdot\left(1-\varepsilon^{-1}\right) \cdot\left(1-\varphi_{a f}+\gamma_{r}\right) \cdot \eta_{v} .
$$

In this expression, $\varphi_{a f}$ quantifies the influence of cylinder additional filling, having the well-known designation of additional filling degree, and $\gamma_{r}$ represents the residual gasses coefficient, being a direct criterion of cylinder residual gasses degree of cleaning evaluation.

In order to simplify the development of the proposed model, it is considered $\varphi_{\text {af }}$ med $\cong$ 0.16 as a positive average and constant value of the additional filling degree, not affected by the engine operating mode changing.

Regarding the dependence between the inlet pressure and engine load calculation, a linear function is introduced in the model:

$$
\eta_{v}(\chi)=\alpha_{n} \cdot \chi+\beta_{n},
$$

which shows, for spark-ignition engines, the filling coefficient variation in case of engine load modification, for different engine speeds.

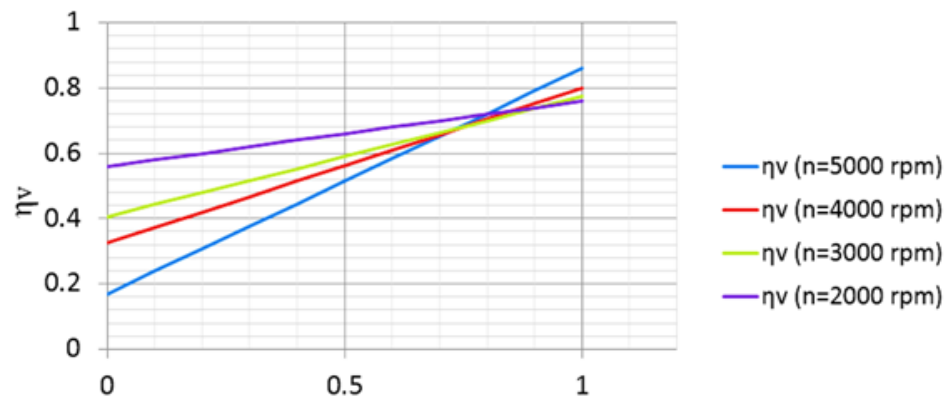

Engine load

Fig. 1. Dependence between filling coefficient and engine load

Establishment of $\alpha_{n}$ and $\beta_{n}$ coefficients from expression (4) was made by analyzing a data series from the literature for $\eta_{v}$, both in terms of, as described above, the maximum corresponding value of the total engine load and the minimum engine load.

The representation of this variation, for an engine speed interval which ensures a stable operationality is shown in Figure 1, as a family of lines with different tendencies.

In this way, for the proposed model it is obtained the mathematically expression which highlights the intake pressure variation together with the engine load modification, for different values of the engine speed: 


$$
p_{a}(\chi)=\Phi_{0} \cdot T_{a} \cdot\left(1-\varepsilon^{-1}\right) \cdot\left(1-\varphi_{a f}+\gamma_{r}\right) \cdot\left(\alpha_{n} \cdot \chi+\beta_{n}\right) .
$$

With $\alpha_{n}, \beta_{n}$ values determined this way, the intake pressure expression $p_{a}(\chi)$ becomes:

$$
p_{a}(\chi)=\Phi_{0} \cdot\left(1-\varepsilon^{-1}\right) \cdot\left(0,84+\gamma_{r}\right) \cdot T_{a}(\chi) \cdot\left\{\begin{array}{ll}
0.69 \cdot \chi+0.17, & n=5 \cdot 10^{3} \\
0.475 \cdot \chi+0.325, & n=4 \cdot 10^{3} \\
0.368 \cdot \chi+0.406, & n=3 \cdot 10^{3}
\end{array} \quad[\mathrm{rpm}] .\right.
$$

Graphical analysis of Figure 1 highlights that for low engine loads, $\eta_{v}$ has high values in low engine speeds domain, the lower rate of airflow contributing on gasodynamic loss lowering. By increasing $\eta_{v}$, the filling duration is contributing in case of small engine speeds, contribution which is higher for these low engine speeds. In case of high engine loads, the gasodynamic resistance is decreasing, irrespective of engine speed value, which leads to $\eta_{v}$ increasing for high engine speeds domain, when the inertia of intake air flow is higher, encouraging in this way the filling. It can be noted a situation of quasi-equilibrium for an engine load of $80 \%$, when $\eta_{v}$ is kept almost constant for all the considered engine speeds.

In expression (6), $T_{a}$ represents the temperature of fresh mixture at the end of intake stroke.

To ensure a better approximation of the theoretical model to the reality, this article proposes an analytical modeling of intake temperature $T_{a}$, depending on the engine load, for different engine speed values. In order to do this, it is considered that $T_{g}$, the temperature of exhaust gasses highlights very accurate the engine operating regimes and therefore the engine thermal regime.

Because of this reason, it is considered that the level and variation of this temperature will influence the heat transfer of the fresh mixture, taking into consideration the engine components surfaces, as intake manifold, cylinder head, pistons, cylinder walls, and also the residual gasses from the combustion chamber, determining considerable the level and evolution of $T_{a}$ temperature of the fresh mixture at the end of the intake process.

On this line, the start consists in adopting a variation of the exhaust gasses temperature, $T_{g}$, dependent on the engine load, by using a quadratic function, whose coefficients are calculated by using collected and processed data experimentally. The results of this processing are summarized in Figure 2.

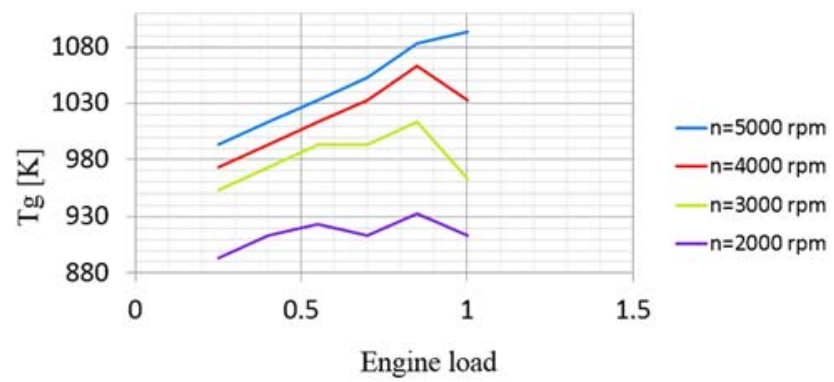

Fig. 2. Exhaust gasses temperature variation dependent of engine load, experimentally determined

The coefficients of this parabolic function of the exhaust gasses temperature, $T_{g}$, were introduced according to the minimum and maximum values of $T_{g}$ temperature and also according to the quadratic function mathematical properties.

By customizing the quadratic function in the present situation, we can define the general function which shows the exhaust gasses temperature variation, $T_{g}(\chi)$, as following: 


$$
T_{g}(\chi)=a_{n_{T_{g}}} \cdot \chi^{2}+b_{n_{T_{g}}} \cdot \chi+c_{n_{T_{g}}},
$$

where $a_{n_{T_{g}}}, b_{n_{T_{g}}}$ și $c_{n_{T_{g}}}$ represents the coefficients of $T_{g}(\chi)$ function.

As already shown in Figure 2 is highlighted the variation of exhaust gasses temperature, $T_{g}$, experimentally obtained, depending on the engine load, for different engine speed values. $T_{g}$ temperature variations for four different engine speed values were mathematic assimilated with four parabolic functions, having the coefficients determined by the method described above.

In these conditions, $T_{g}(\chi)$ function is expressed as following:

$$
T_{g}(\chi)=\left\{\begin{array}{ll}
-278 \cdot \chi^{2}+473 \cdot \chi+893, & n=5 \cdot 10^{3} \\
-167 \cdot \chi^{2}+284 \cdot \chi+913, & n=4 \cdot 10^{3} \\
-28 \cdot \chi^{2}+48 \cdot \chi+943, & n=3 \cdot 10^{3} \\
-56 \cdot \chi^{2}+95 \cdot \chi+873, & n=2 \cdot 10^{3}
\end{array} \quad[\mathrm{rpm}] .\right.
$$

The graphical representation of the exhaust gasses temperature, by considering the parabolic function $T_{g}(\chi)$ analytically determined is highlighted in Figure 3.

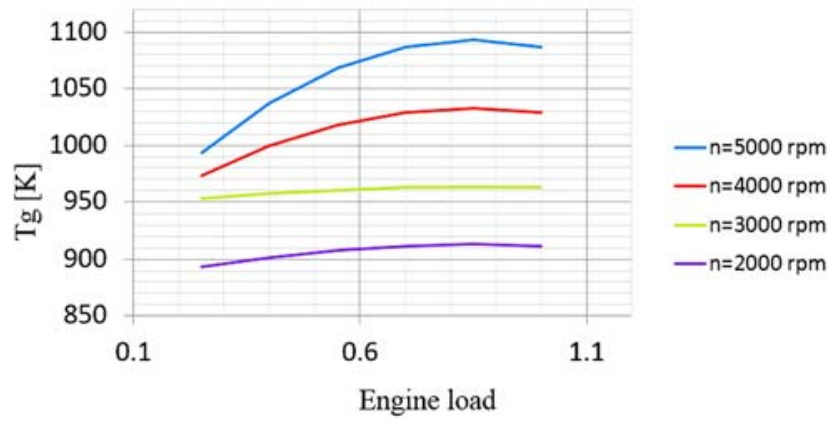

Fig. 3. Parabolic variation of exhaust gasses temperature $T_{g}$ depending on the engine load

Based on the reasoning exposed above, sill considering for $T_{a}$ temperature the same type of variation, we can write the analytical expression used to determine the variation of temperature depending on engine load, for different engine speed values $T_{a}(\chi)$ :

$$
T_{a}(\chi)=a_{n_{T_{g a}}} \cdot \chi^{2}+b_{n_{T_{a}}} \cdot \chi+c_{n_{T_{a}}} .
$$

Considering the evolutions of temperatures $T_{a}$ and $T_{g}$ with the same difference $\Delta T=$ $T_{\max }-T_{\min }$ and adopting values for $T_{a_{\min }}$ temperature, corresponding to the minimum engine load, on every engine speed value, the coefficients of the function were determined, according to expression (10). Using this coefficients, function $T_{a}(\chi)$ becomes:

$$
T_{a}(\chi)=\left\{\begin{array}{ll}
-278 \cdot \chi^{2}+473 \cdot \chi+225, & n=5 \cdot 10^{3} \\
-167 \cdot \chi^{2}+284 \cdot \chi+263, & n=4 \cdot 10^{3} \\
-28 \cdot \chi^{2}+48 \cdot \chi+312, & n=3 \cdot 10^{3} \\
-56 \cdot \chi^{2}+95 \cdot \chi+299, & n=2 \cdot 10^{3}
\end{array} \quad[\mathrm{rpm}] .\right.
$$

In this conditions, the expression of $p_{a}(\chi)$ becomes: 


$$
\begin{gathered}
p_{a}(\chi)=\Phi_{0} \cdot\left(1-\varepsilon^{-1}\right) \cdot\left(0.84+\gamma_{r}\right) \cdot\left\{\begin{array}{l}
-278 \cdot \chi^{2}+473 \cdot \chi+225 \\
-167 \cdot \chi^{2}+284 \cdot \chi+263 \\
-28 \cdot \chi^{2}+48 \cdot \chi+312 \\
-56 \cdot \chi^{2}+95 \cdot \chi+299
\end{array}\right. \\
\left\{\begin{array}{ll}
0.69 \cdot \chi+0.17, & n=5 \cdot 10^{3} \\
0.475 \cdot \chi+0.325, & n=4 \cdot 10^{3} \\
0.368 \cdot \chi+0.406, & n=3 \cdot 10^{3} \\
0.2 \cdot \chi+0.56, & n=2 \cdot 10^{3}
\end{array}[\mathrm{rpm}]\right.
\end{gathered}
$$

Coefficient $\gamma_{r}$, direct and particular criterion to estimate the quality of purification of the engine cylinder by the residual gasses is defined by the following expression:

$$
\gamma_{r}=\frac{M_{r}}{M_{a}}
$$

where: $M_{r}$ - quantity of residual gasses [kmol], $M_{a}$ - quantity of fresh mixture [kmol].

The influence of the functional factors on the residual gasses coefficient is complex, the proposed model being focused on this aspect. In this way, the modification of this coefficient depending on the engine speed value has a minimum contribution $[2,4]$.

A second influence is given by the engine load. Thus it was demonstrated experimentally that increasing the engine load implicitly leads to an increase of the residual gasses quantity, which remains inside the engine cylinder after the evacuation process. On the other hand, as highlighted by some authors, it may be noted a sharp increase of the residual gasses coefficient $\gamma_{r}$ while the spark-ignition engine's load is reduced. In the same time, the residual gasses acts in a chemical way, by introducing a kinetic consequence, because being chemically inert substances, leads to a reduced fuel burn rate. This inhibitor action interferes with a large share of the spark-ignition engines, in partially load regimes.

Based on this brief related analysis, in this article developed by the authors a variation of residual gasses coefficient, depending only on the engine load is adopted, considering that the variation depending on the engine speed is insignificant. Considering this analysis, and also data from the literature, a variation depending on the engine load according to the following expression is established:

$$
\gamma_{r}(\chi)=a_{\gamma_{r}} \cdot \chi+b_{\gamma_{r}}
$$

in which the coefficients of this function are: $a_{\gamma_{r}}=-0.3$ și $\mathrm{b}_{\gamma_{\mathrm{r}}}=0.35$. In this situation, function $\gamma_{r}(\chi)$ becomes:

$$
\gamma_{r}(\chi)=-0.3 \cdot \chi+0.35
$$

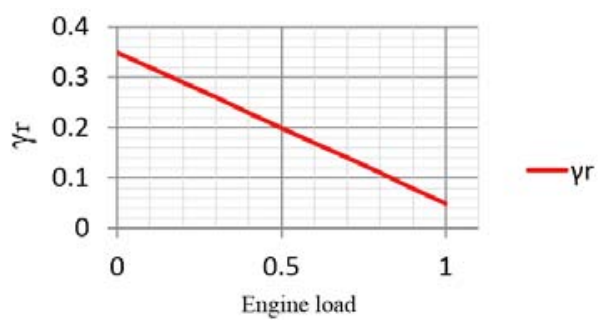

Fig. 4. Variation of residual gasses coefficient depending on the engine load

Finally, by using these functions, we can define the $\prod$ parameters at the end of the intake process, obtained through this developed model: 


$$
\prod:\left\{\begin{aligned}
& T_{a}(\chi)=\left\{\begin{array}{ll}
-278 \cdot \chi^{2}+473 \cdot \chi+225, & n=5 \cdot 10^{3} \\
-167 \cdot \chi^{2}+284 \cdot \chi+263, & n=4 \cdot 10^{3} \\
-28 \cdot \chi^{2}+48 \cdot \chi+312, & n=3 \cdot 10^{3} \\
-56 \cdot \chi^{2}+95 \cdot \chi+299, & n=2 \cdot 10^{3}
\end{array} \quad[\mathrm{rpm}]\right. \\
& p_{a}(\chi)=\Phi_{0} \cdot\left(1-\varepsilon^{-1}\right) \cdot[0,84+(-0,3 \cdot \chi+0,35)] \cdot \begin{cases}-278 \cdot \chi^{2}+473 \cdot \chi+225 \\
-167 \cdot \chi^{2}+284 \cdot \chi+263 \\
-28 \cdot \chi^{2}+48 \cdot \chi+312 \\
-56 \cdot \chi^{2}+95 \cdot \chi+299\end{cases}
\end{aligned}\right.
$$

\section{Theoretical results. Simulations}

Based on these simulations, the variation of temperature $T_{a}$ and pressure $p_{a}$ were highlighted, depending on the engine load, for different engine speed values, as shown in Figure 5 and Figure 6 . In this way, the parameters which establish the engine performance can be easily obtained, especially the fuel consumptions of the engine.

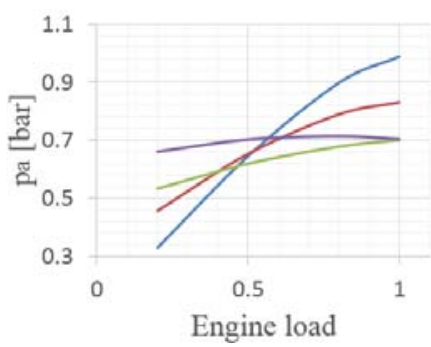

Fig. 5. Intake pressure $p_{a}$ variation dependent of engine load

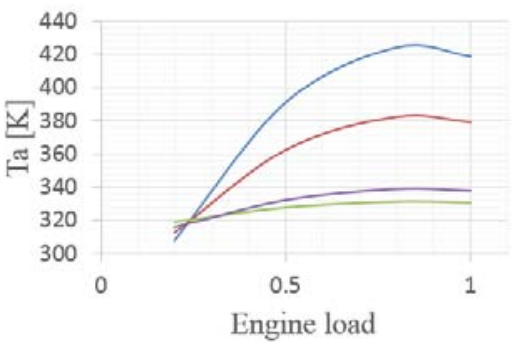

Fig. 6. Intake temperature $T_{a}$ variation dependent of engine load

\section{Conclusions}

It is estimated that the developed model allows a performance evaluation and a behavior study of the spark-ignition engines, used in automotive propulsion. In this way, a contribution in the cooperation between the engine and the vehicle optimization can be offered, still in the design phase, shortening the introduction of the manufacturing cycle for new models. The result of this study will lead in the end to cost reductions.

\section{References}

1. V. Raimbault et al., 16. Internationales Stuttgarter Symposium, 721-735 (Springer, 2016)

2. B. Grünwald, Theory, calculation and construction of motor vehicles engines (in Romanian), (EDP, București, 1980)

3. S. Buhl et al., Flow Turbul Combust J. 98 (2), 579-600 (2017)

4. D. M. Dogariu at al., CONAT 2016 International Congress of Automotive and Transport Engineering, 187-194 (Springer, 2016) 